\title{
La discursivización de la incertidumbre espacial en Desde el cielo de Manuel Murguía ${ }^{1}$
}

\section{Arantxa Fuentes Ríos}

\section{Alba Rozas Arceo \\ Universidade de Santiago de Compostela}

Resumen: El presente estudio pretende trazar un análisis de la incertidumbre espacial como fundamento de la ficción en la novela Desde el cielo de Manuel Murguía. Con este propósito, llevaremos a cabo, en primer lugar, una aproximación a la problemática que rodea la definición del paradigma de la incertidumbre espacial, así como su representación. A continuación, estudiaremos la obra atendiendo particularmente a la caracterización vaga e imprecisa de los enclaves en los que se desarrolla el argumento. Finalmente, a partir de la metodología que provee la cartografía literaria y las técnicas de análisis que facilitan los Sistemas de Información Geográfica, desarrollaremos una tipología que permitirá dirimir la incertidumbre espacial del discurso narrativo y relacionar los espacios ficcionales de la novela con sus referentes reales.

Palabras clave: incertidumbre espacial, Sistemas de Información Geográfica, Santiago de Compostela, Manuel Murguía

Résumé: Notre travail cherche à approfondir le concept d'incertitude spatiale, partant d'une étude de cas, le roman Desde el cielo de Manuel Murguía. Premièrement, nous nous attarderons sur les difficultés qui entourent la notion d'incertitude, de même que les défis de sa représentation cartographique. Deuxièmement, nous aborderons le traitement spatial de l'œuvre de Murguía, caractérisé par une peinture diffuse et imprécise des lieux. Finalement, nous proposerons une typologie qui cherche à éclaircir les limites de l'incertitude spatiale. 
Mots-clés: incertitude spatiale, GIS, Saint-Jacques de Compostelle, Manuel Murguía

El tratamiento de la incertidumbre espacial en los textos ficcionales constituye uno de los desafíos más complejos de la cartografía literaria. Dentro de esta disciplina, la incertidumbre se ha consolidado como un paradigma de plena actualidad sujeto a grandes controversias que derivan de su conceptualización. En la línea de lo que han observado Buttenfield (1993), MacEachren et al. (2005) o Drecki (2007), dicha noción remite a la imprecisión con que a menudo se utiliza la información espacial en relación a la realidad empírica. Asimismo, la incertidumbre espacial plantea serios problemas de representación dentro de las disciplinas vinculadas a los Sistemas de Información Geográfica, a pesar de las múltiples investigaciones centradas en su descripción y acotación (Griethe/Schumaan 2006).

De acuerdo con las perspectivas de análisis que favorece la cartografía literaria y a partir de un estudio de caso, la novela de Manuel Murguía Desde el cielo (1854), en estas páginas propondremos una delimitación del concepto de incertidumbre espacial, abordando la ambigüedad de los datos espaciales en la ficción y sus posibilidades de determinación geográfica. Desde el punto de vista metodológico, aplicaremos diferentes mecanismos de análisis que proveen los Sistemas de Información Geográfica.

El estudio de esta novela resulta de especial interés, pues constituye un ejemplo paradigmático del innato diálogo entre precisión e indeterminación del espacio en la literatura. Nos situamos en un terreno propicio a la evocación y la sugerencia, donde surgen lagunas de información, ya sean espaciales o no, y que transcurren en el pacto discursivo. Dentro de esta narración, corresponderá al lector explorar los cauces de la imprecisión del texto y al investigador delimitar el alcance de la incertidumbre espacial de la ficción.

\section{Los desafíos de la incertidumbre espacial}

La incertidumbre espacial deviene un campo específico dentro del discurso ficcional, que incluso puede ser tratado al margen de la representación cartográfica. Dicho paradigma 
discurre por líneas muy concretas que exigen ser correctamente conceptualizadas. Con el propósito de delimitar la especificidad de la incertidumbre espacial que caracteriza la ficción de Desde el cielo se revela imprescindible llevar a cabo, en primer lugar, una revisión de este concepto que subraye su potencial semántico y, posteriormente, analizar su funcionalidad y materialización en la novela estudiada.

La incertidumbre espacial constituye una de las cuestiones más espinosas a las que se enfrenta la cartografía literaria y ha ocupado a teóricos de muy diversos campos, no siempre estrictamente literarios. Destacan sobre todo especialistas vinculados al ámbito de las ciencias de información geográfica, entre los que sobresalen MacEachren et al. (2005), Thomson et al. (2005) o Drecki (2007).

La primera dificultad a la hora de definir la incertidumbre espacial atañe a la falta de un significado unívoco y aceptado de manera general para este concepto. Desde los más tempranos intentos de determinar la idea de incertidumbre espacial, distintos especialistas proceden estableciendo categorías. En consecuencia, surgen nociones como error, vacilación, aleatoriedad, vaguedad, precisión o imprecisión a las que se pretende atribuir, de manera teórica, características particulares, tal como han procurado, entre otros, Zhang y Goodchild (2002: 6-7), MacEachren et al. (2005: 140) o Thomson et al. (2005). No obstante, y para mayor dificultad, en la práctica, estos componentes que se utilizan para tratar de definir el concepto de incertidumbre espacial son a menudo intercambiables (Drecki 2007).

Sin embargo, las diferentes tentativas de delimitar el concepto de incertidumbre espacial también ponen de manifiesto, al margen de la complejidad y grado de descomposición de sus elementos, una apreciación general que tiene que ver con la carencia de información con respecto a los datos geográficos, tal como señalan Thomson et al. (2005). Por tanto, al margen de un análisis de las particularidades que pueden presentar los datos geográficos, es posible concluir que la carencia u omisión de información constituye una de las características fundamentales de la incertidumbre espacial. Esta variable actúa de manera específica en el caso de los textos literarios.

De este modo, a la hora de establecer una definición de la incertidumbre espacial que opera 
en Desde el cielo, es necesario tener en consideración, tal como señalan Griethe y Schumann (2006), que este paradigma representa: "a unified term that subsumes the relevant kinds of distrust in data". Este déficit de información implica en muchos casos una diversidad o especificidad que, siguiendo la línea de Griethe y Schumann, requiere un tratamiento concreto.

\section{El tratamiento espacial en Desde el cielo de Manuel Murguía}

Desde el cielo, novela de juventud de Manuel Murguía², fue divulgada inicialmente por entregas en la revista La Iberia en 1854, cuando el autor contaba tan solo con diecisiete años. Décadas más tarde, siendo ya un intelectual reconocido, la obra se publicó en 1910. Este relato breve orbita alrededor de un malogrado triángulo amoroso entre los protagonistas: Marta, Juanito y Blanca. La acción transcurre fundamentalmente entre dos ejes espaciales: la ciudad de Santiago de Compostela y la zona de Padrón, que el narrador denomina "P", marcada por el curso del río Ulla y Sar. La trama destaca por su sencillez. Amigos de la infancia y jóvenes enamorados, Marta y Juanito viven su relación en la tranquila villa de "P" hasta que Juanito debe marchar a Santiago de Compostela para iniciar sus estudios. Allí encontrará fortuitamente a una bella dama llamada Blanca que le hará olvidar su compromiso inicial con Marta. Tiempo más tarde Juanito regresará a "P" para casarse con Blanca, lo que abocará a Marta al suicidio. La tragedia final se sucede tras una grave inundación que hace desbordar el Sar y el Ulla y que acabará con la vida de la pareja de recién casados.

Desde el cielo presenta escasos referentes espaciales pero de gran simbología, en este caso los ríos Sar y Ulla, la ría de Arousa y la ciudad de Santiago de Compostela. A nivel espacial domina, a excepción de ciertos espacios significativos, un tipo específico de incertidumbre espacial, como explicaremos a continuación.

\subsection{El trazado de la incertidumbre espacial en Desde el cielo}

En el caso particular de la novela Desde el cielo es posible constatar una tendencia muy clara del autor a procurar la ambigüedad, la imprecisión y la vaguedad espacial. Con 
respecto a esta última apreciación, resultan útiles ciertas aportaciones que ahondan en las causas de las lagunas de información geográfica generadoras de incertidumbre.

En concreto, la novela elegida constituye un ejemplo paradigmático de que, tal como observan Zhang y Goodchild (2002: 5), la incertidumbre especial: "is not a property of the data contents so much as a function of the relationships between data and users. In this context uncertainty may be defined as a measure of the difference between the data and the meaning attached to the data by the current user (...) by direct and perfectly accurate observation of reality".

Siguiendo esta línea, la incertidumbre espacial en esta obra deriva de la falta de concreción o insuficiencia de información geográfica que el autor proporciona de manera intencionada. En cuanto entra en juego el conocimiento del lector - y del investigadorsobre la realidad geográfica representada, es posible atribuir un referente concreto a los principales escenarios recreados en la narración. Por tanto, se trata de un tipo de incertidumbre que atañe a la representación textual de los datos geográficos, la cual, de acuerdo con los postulados de la cartografía literaria, deriva del propio lenguaje literario y de la libertad artística del escritor (Reuschel/Hurni 2011: 298).

La incertidumbre que atañe a los conceptos lingüísticos es connatural a las localizaciones en las que transcurre la ficción, porque, tal y como afirma Reuschel/Hurni (ibídem), constituyen: "an interpretation of reality, decorated and even camouflaged through the imagination of the author and made accessible by written words and by natural language". En otras palabras, las localizaciones ficcionales no resultan simples imitaciones del espacio empírico, sino que el discurso literario en general, y esta novela en particular, se caracteriza por un mayor o menor grado de ambigëdad y vaguedad, connatural al lenguaje (Auger/Roy 2008: 1861).

No obstante, es preciso señalar que los datos espaciales de Desde el cielo pueden someterse a un proceso de desambiguación. Tal y como veremos, en esta novela la incertidumbre espacial resulta consustancial al lenguaje ya que, como señalan Waters y Evans (2003): 
The vast majority of people don't use a scientific geographical vocabulary, nevertheless most use a wide variety of geographical terms on a day to day basis (...) a vernacular geographical terminology which is vastly more used than the coordinate systems and scientifically defined variables.

Esta premisa no impide delimitar los espacios literarios en los que se recrea, contraponiendo la información espacial del texto con la realidad. Es más, la representación del espacio en Desde el cielo se corresponde con, siguiendo a Waters/Evans (2003), "particular geographical areas, but without a clear definition of where or what they are". Este tratamiento ficcional se vincula a la inserción de la obra en el género romántico de la época, proclive a espacios medievalizantes, leyendas ancestrales y personajes trágicos. Esta modalidad narrativa se caracteriza por el predominio del lenguaje poético, la escasa trama narrativa, la discontinuidad tanto espacial como temporal o la focalización en la vida interior de los personajes.

Los teóricos Shu et al. (2003: 4) clasifican la falta de información que deriva en incertidumbre espacial según provenga de datos puramente geográficos, temáticos o temporales. En la obra estudiada se produce una conjunción de estos tres factores, si bien el mayor grado de inconcreción se detecta en el espacio y el tiempo. El ritmo temporal lento y pausado de la novela, propio del aire mítico subyacente a la historia, redunda en la atemporalidad característica de la leyenda romántica que influye asimismo en el tratamiento del espacio. Esta vaguedad espacial y temporal será de nuevo protagonista décadas más tarde en lo que la crítica ha denominado novela lírica.

En consonancia con el género romántico al que pertenece la novela, Desde el cielo se caracteriza por un predominio de la incertidumbre espacial. Una primera modalidad de este tratamiento espacial es la discontinuidad de los escenarios en la novela, entendida como el desarrollo de la acción a partir de una serie de lugares concretos que se interpolan con otros espacios imprecisos, sin hacer explícitas las transiciones de los primeros a los segundos. Esta discontinuidad espacial se refuerza a nivel temático, en virtud de la vaguedad lírica propia al estilo de la novela. Por último, el aire atemporal de leyenda de la novela contribuye a la presentación del espacio en términos genéricos como reducto 
natural, con un marcado carácter idílico, rehuyendo en numerosas ocasiones de las alusiones a escenarios específicos para la acción.

En definitiva, a partir de escasas referencias espaciales precisas y, por tanto, identificables en un mapa, el narrador dibuja profusas descripciones en las que la naturaleza juega un papel clave. El protagonismo del paisaje, típico de la novela romántica, se une a la querencia de Murguía por el espacio gallego, tejiendo así una atmósfera atemporal donde las vegas de los ríos, el discurrir del agua y los paisajes forman un organismo vivo que conducirá la trama hasta su desenlace final.

De este modo, la incertidumbre espacial presente en Desde el cielo deriva del discurso ficcionalizado, a través del cual el autor pone de manifiesto su conocimiento, percepción y dominio de las técnicas de composición literaria propias del romanticismo. Estas se traducen, desde una perspectiva cartográfica, en lo que Egenhofer/Mark (1995: 4) denomina: "the way people think and reason about geographic space and time". Por esta razón, tanto la novela romántica como la novela lírica se presentan como un terreno de potencial interés para el estudio de la incertidumbre espacial.

Antes de establecer una tipología que permita concretar geográficamente los diferentes tipos de incertidumbre discursiva desarrollados en la novela, conviene llevar a cabo una aproximación preliminar a los diferentes espacios concretos en los que se desarrolla la novela, dado que contienen las claves del tratamiento particular que confiere Murguía a la información geográfica en la ficción.

\section{El enclave geográfico en Desde el cielo: Padrón y Santiago}

La novela transcurre en dos espacios completamente antagónicos, unidos por el río Sar. Por un lado se encuentra la villa de "P", lugar de origen de los tres protagonistas. A pesar de nombrarla únicamente a partir de su inicial, el topónimo resulta fácilmente reconocible para el lector gallego o conocedor de las Rías Baixas. El paso del río Ulla, la presencia del Sar y la cercanía a la ría de Arousa hace que la "P" sea identificable con Padrón. Si además se introduce el relevante dato de las inundaciones, tan recurrentes en la 
época, este espacio potencialmente incierto en el discurso en realidad resulta transparente a nivel geográfico para los lectores familiarizados con este contexto.

Es importante señalar que este recurso de incertidumbre lingüística es una de las características reseñables del usus escribendi del autor. Murguía juega en otras ocasiones a lo largo de la novela con introducir únicamente la inicial de topónimos o antropónimos, como sucede con "el marqués de B", o incluso no concreta fechas como "el mes de febrero de 184... ". Se trata de un recurso estético propio de la época y al que Murguía recurre también en otras ocasiones, como en su obra La primera luz (1860), que firma como "M... ". Debido a esta cierta transparencia semántica, estos recursos no se traducen en una incertidumbre constatable, sino que entroncan con la vaguedad propia del discurso de la evocación lírica.

La villa de "P" se describe a partir de la naturaleza circundante, espacio aparentemente idílico pero que puede tornar súbitamente en tragedia debido a la fuerza destructora del agua. El río Sar actúa como hilo conductor entre esta localización y la ciudad de Santiago. Caracterizado por oposición a la villa, Santiago es el lugar en el que Juanito se enamora de Blanca y olvida a Marta. En la novela aparece representada como una ciudad gris, "triste", cubierta de "cielo ceniciento", sobre la que destacan las torres de la catedral, que proyectan sus sombras sobre toda su extensión: "Santiago es la ciudad sombría, la ciudad monumental que esconde sus cien torres de granito entre las nieblas que prenden y envuelven la cima de los montes que la rodean y ahogan con sus brazos descarnados y con sus frías caricias" (Murguía 1910: 31).

Juanito y Blanca se definen por ser personajes activos, dueños de sus propias vidas, que se desplazan por diferentes espacios: de Santiago a "P", de Santiago a Conxo o de la catedral al palacio en el que vive Blanca. Ambos deciden casarse, retornar a "P" e iniciar allí su vida en pareja, atraídos por su naturaleza apacible. A este propósito el narrador apunta: "Allí serían más felices, nadie les estorbaría; allí había lagos y ríos de crecida corriente, y de orillas frescas y sombrías en donde pasar las más hermosas horas de la vida" (idem: 64).

Mientras que Santiago es el espacio únicamente compartido por Blanca y Juanito, Marta aparece en perfecto sincretismo con el paisaje que rodea a "P". Testigo del dolor de la 
joven, los malos augurios se manifiestan ya desde la partida de Juanito, originando la primera inundación de la novela. Este tipo de premoniciones, características de la imaginería popular, muestran a Marta en plena simbiosis con la naturaleza:

Cuando se retiró de la ventana, la tempestad que se aproximaba, estalló con ímpetu; rasgáronse las nubes, la lluvia inundó la tierra, alzáronse las olas del mar y el Sar, abandonando su lecho de flores, engrosó su corriente e inundó la campiña con sus aguas de color verdoso. (idem: 29)

Marta vive presa en su ensimismamiento, confinada en su casa y recluida en su música. Su actitud se rompe con la decisión trágica del suicidio. Se trata de un personaje prototípicamente romántico, atormentada por los sueños y los malos augurios. La intensificación de la atmósfera propia de la novela romántica se materializa en ella, reflejo de toda la carga sensorial que emana del fluir de las aguas y la música de Schumberg.

Esta caracterización del personaje determina la relevancia que adquiere en la novela el espacio natural, recreado en ríos, lagunas, orillas, montes, bosques o lagos. Todos estos elementos orográficos componen una cartografía donde el paisaje prototípicamente gallego se convierte en protagonista indiscutible de la historia. Santiago de Compostela, ciudad emblemática y de gran calado literario, queda difuminada al lado de la fuerza que adquieren los ríos Sar y Ulla, así como sus parajes, a lo largo de la trama narrativa. En relación a este punto debemos subrayar que buena parte de la incertidumbre que caracteriza la narración proviene de la dificultad de ubicar sin la ayuda cartográfica los espacios naturales que se van sucediendo en la obra. Además de su indudable hegemonía, los enclaves relacionados directamente con el Sar y el Ulla gozan de una señalada recurrencia, intensificando así su presencia.

No resulta difícil identificar la inicial de "P" con la villa de Padrón. Padrón y Santiago, espacios compartidos por Murguía y Rosalía de Castro, son el trasfondo de un rico diálogo intertextual que se establece entre ambos autores. El poema “Torres d' Oeste” de Rosalía de Castro, incluido en Follas Novas (1880) su novela Flavio (1861), así como diversos textos de Rosalía dedicados a Padrón se ven iluminados desde el prisma de Desde el cielo. Las correspondencias comienzan ya en las percepciones sugeridas por los espacios dicotómicos 
de Santiago y Padrón. De nuevo, Santiago aparece como un lugar proclive a la melancolía y la tristeza. Rosalía de Castro (1993: 2, 631) afirma en su artículo titulado "Padrón y las inundaciones" (1881) que a Padrón se llega "huyendo al eterno clamoreo de las campanas de Compostela, cuyos ecos, mezclados a los bramidos de las tempestades invernales, parecen perseguir con saña los ánimos entristecidos".

Los parajes de Padrón, rodeados del Sar, el Ulla y la ría, inspiran belleza al tiempo que provocan inquietud. Se trata de un espacio bucólico que súbitamente torna en naturaleza devastadora. En Desde el cielo la inundación final viene provocada por el suicidio de Marta, tirándose a las aguas del Ulla cerca de la villa de "P". El segundo día de la boda de Blanca y Juanito amanecerá cubierto de malos augurios, remordimientos y "hasta avisos del Cielo". La tempestad entra con la llegada de la noche:

El Sar gemía y se engrosaba; el Ulla, que le recibía en sus brazos, abandonaba ya su lecho de flores y arbustos para entrar en el Océano, que se adelantaba a recibirle, y la luna, oculta entre las nubes, negaba a la tierra su luz suavísima y apacible. (idem: 88-89)

Al rugido del mar le siguieron "gritos humanos, dolientes, agudos"; un panorama de destrucción y muerte que acaba con los dos cuerpos de Juanito y Marta entrelazados. El tono legendario del relato culmina en la tragedia final, donde convergen todos los hilos narrativos.

También en este sentido, el relato resulta verosímil con la referencia geográfica de Padrón. Las inundaciones en la villa constituían un hecho sobradamente conocido por la población, como da cuenta el abundante material gráfico de la época. Ya Murguía en el V capítulo de su célebre Galicia (1888) se refiere al gran lago que en otro tiempo rodeaba Padrón. La recurrencia de las inundaciones y la fuerza destructora de las mismas originó diversas leyendas. La propia Rosalía de Castro en su ya mencionado artículo "Padrón y las inundaciones", publicado en 1881 en La Ilustración Gallega, a que "cuéntase" que la colegiata de Santa María quedó anegada bajo las aguas de una terrible inundación. Del mismo modo que recogía el terror causado por las inundaciones: "El ensordecido Ulla 
cegaba casi los arcos del puente y, amenazando tragarse la parte baja de la población, lo inunda todo en derredor, silencioso como la muerte" (ídem: 663).

Tanto en Murguía como en Rosalía el río, el sonido del agua, ejerce en sus personajes prototípicamente románticos una atracción fatídica. No solo Marta llega al Ulla y escoge suicidarse, sino que también son las aguas de un río en las que el protagonista de Flavio busca su propia muerte. El agua ejerce una atracción fatídica en Marta, que sale de su habitual ensimismamiento para lanzarse a las aguas "loca y fuera de sí" (idem: 77). Algo similar le sucede al yo lírico del poema rosaliano "Torres d'Oeste" perteneciente a Follas Novas (2004: 367) donde una "negra tentazón" que le "anubrou a vista" le condujo siniestramente a buscar la muerte tirándose al agua.

\section{Tipología de la incerteza discursiva en Desde el cielo}

Ante la especificidad que presentan los escenarios de esta novela, resulta necesario establecer una clasificación de espacios que permita distinguir, en función de los procedimientos cartográficos necesarios para su detección, los diferentes tipos de incertidumbre espacial presentes en el texto narrativo.

Desde la perspectiva de la cartografía literaria, el principio que sustente la tipología de la incertidumbre espacial en Desde el cielo debe ser, siguiendo a Thomson et al. (2005), "to identify the broad categories for uncertainty and determine how to identify and model the specific instances of those types of uncertainty in a particular domain". En este sentido, no debemos olvidar que parte de la incertidumbre discursiva puede ser dirimida por el analista y su capacidad de asociar, interpretar y delimitar los datos de los que dispone a través del cotejo con el espacio empírico que favorecen las tecnologías de la información geográfica. La tarea del especialista consistirá en utilizar las herramientas y metodología que provee la cartografía literaria para cubrir lagunas de información espacial que presenta la narración, con el propósito de tejer el entramado de localizaciones que la sustenta. Con este objetivo, es posible establecer una tipología de espacios que permite reducir la imprecisión y ambigüedad textual a la precisión geográfica, de acuerdo con un planteamiento gradual que parte de los elementos localizables hasta los inciertos. 
De este modo, en un primer nivel de análisis se sitúan los espacios precisos, esto es, aquellas localizaciones cuya descripción o denominación en el texto remite de manera directa o latente a un referente geográfico real. En esta línea se enmarcan las alusiones a Padrón y Santiago de Compostela, el río Sar o el Ulla.

El ámbito de la incertidumbre discursiva corresponde a los espacios asignados, a saber, espacios cuyo referente real, eludido en la ficción, puede ser determinado por el investigador a partir de la interpretación del texto y los datos geográficos del territorio empírico.

Dentro de esta categoría discerniremos dos subclases: asignado de grado 1 y asignado de grado 2 . En el primer grupo se encuentran aquellos espacios, cuya ubicación no aparece especificada en la narración, pero a los que el analista puede atribuir una localización real concreta a partir del estudio de los datos textuales y de la información contextual sobre el autor, la obra y el escenario en el que se ambienta. Por el contrario, la localización de los espacios asignados de grado 2, requiere el empleo de recursos específicos de las técnicas de representación geográfica a la hora de adscribir unas coordenadas reales concretas a un enclave cuya ubicación textual resulta ambigua. De este modo, múltiples datos, que en la narración se presentan como inciertos, pasan a ser asignables tras la revisión cartográfica del escenario. Esta distinción pone de manifiesto que las tecnologías propias de los Sistemas de Información Geográfica no constituyen un mero instrumento de visualización, sino que proporcionan una metodología determinante a la hora de distinguir la incertidumbre del espacio textual de la realidad geográfica.

Por último, se encuentran los espacios dependientes, donde la aparente incertidumbre espacial respecto a su ubicación geográfica real queda resuelta a partir de la relación directa que se establece en el texto con otros espacios precisos o asignados. En esta clase de referencias, la ambigüedad discursiva se resuelve a partir del propio contexto lingüístico (Auger/Roy, 2009: 1864). De este modo, la localización de un espacio determina la ubicación de otro lugar vinculado a él.

En este sentido, es preciso subrayar que esta tipología busca detectar la incertidumbre discursiva. El análisis del texto y de su cartografía permite aclarar las 
referencias imprecisas, aunque transparentes en diverso grado para los especialistas y también para los lectores de la época.

Veamos ahora este planteamiento tipológico aplicado al texto de Murguía. A partir del estrecho vínculo que establece Murguía con el lector competente, es posible determinar las claves que subyacen a la discursivización de la incerteza en la novela. Por un lado, la topografía que predomina en la novela no es descriptiva sino profundamente evocadora, procedimiento que obedece a la intencionalidad de gestar un paisaje de gran fuerza lírica. A excepción de Santiago de Compostela y los ríos Sar y Ulla, el resto de las referencias espaciales destacan por su alto grado de incertidumbre, aunque con distinto tratamiento. Esta marcada indefinición referencial requiere el trazado de una primera cartografía de escenarios que se corresponda con el discurso topográfico característico de la narración romántica. La complejidad de este tipo de representación deriva del protagonismo que adquiere la naturaleza, en forma de ríos, márgenes, bosques, colinas, marinas o lagos. A través de estos elementos espaciales Murguía construye un paisaje de profundas connotaciones poéticas, donde resulta difícil discernir la presencia de referentes reales frente a descripciones prototípicas de un espacio idílico propicio para el amor y la tragedia. Los lugares claramente definidos se limitan a Santiago de Compostela y su catedral, Padrón y los ríos Ulla y Sar, que dentro de nuestra tipología se denominan precisos. A partir de estos topónimos se despliegan una larga serie de enclaves que en su mayoría responden a la categoría de asignables. Puesto que estamos ante una presencia muy marcada de una naturaleza idealizada, con la imprecisión que en sí misma implica, los espacios dependientes no adquieren una presencia significativa en la novela, de ahí que no sean analizados.

El mayor reto de nuestra tipología parte la distinción entre asignable de primer grado y asignable de segundo grado. Como ya explicamos, el primer tipo no requiere la utilización de mecanismos de análisis exclusivos de los Sistemas de Información Geográfica para relacionar la localización literaria con su referente real. Un ejemplo de espacio asignable en primer grado lo encontramos en el capítulo VII (idem: 53): 
Hay lejos de la ciudad un antiguo monasterio que otro poeta ha comparado ya a las viejas abadías de Bretaña. En su sencilla fachada, en sus torres de piedra, en los árboles que sombrean un largo campo tendido a sus pies, en su puerta entornada, en el rayo de sol que la ilumina, en el silencio que tiende sus alas por el claustro solitario, en aquellos pájaros que huyen al ruido de vuestros pasos...En aquella frente cuyo raudal cae con sonido monótono sobre la taza de granito medio cubierta de musgo; en aquel misterio, en fin, que rodea el edificio de otras épocas, y hallaría el poeta el germen de sus melancólicas meditaciones.

Las referencias del narrador a la majestuosidad del edificio, la presencia del "viejo claustro" (ibidem), así como su proximidad a un "pequeño barrio pobre y sucio" (ibidem) y a un bosque, evoca con cierta transparencia el monasterio de Conxo, espacio emblemático en la ciudad de Santiago. Fernando Cabo (2016), en su estudio a propósito de la novela El primer loco de Rosalía de Castro, subraya el trasfondo histórico-legendario de Conxo, al tiempo que recorre la historia del monasterio que desembocó en su conversión a finales del siglo XIX en un hospital psiquiátrico. Cabo analiza el rico juego intertextual que se establece entre el capítulo que Neira de Mosquera dedica a este espacio en su Monografía de Santiago (1850), la deuda del propio Murguía con este texto, para finalmente analizar la profunda relación intertextual existente entre Desde el cielo y la novela El primer loco de Rosalía de Castro. Entramos así en un rico juego intertextual que ya avanzamos previamente.

En efecto, el santuario de Conxo resulta un espacio representativo del trasfondo legendario y atemporal que Murguía impregna a la novela y que conecta con el gusto romántico. No debe pasar inadvertida la evocación inicial a "las viejas abadías de Bretaña". Esta referencia trasciende la dimensión legendaria al establecer un paralelismo entre ambos paisajes, lo cual conduce a la teoría del celtismo y del atlantismo, de la que Murguía fue uno de sus principales defensores ${ }^{3}$. Asistimos así a un incipiente proceso de discursivización de Conxo como espacio literario y representativo de la toponomia santiaguesa.

Sin duda alguna, los espacios que presentan mayores dificultades a la hora de dilucidar su referente geográfico real son los que han sido tipificados como asignables de segundo grado, debido a su condición, $a$ priori, cuasi fronteriza con los enclaves 
ilocalizables. Esta variable exige un complejo estudio, a la par que arriesgado, pues la adscripción de dichos espacios a su referente real requiere la aplicación de técnicas de análisis cartográfico en función de los datos espaciales extraídos del propio texto, el estudio de documentación diversa relacionada con el texto y el registro de espacios urbanos y naturales recogido a través de las tecnologías GIS. De este modo, se puede reconocer espacios ciertos inciertos en la narración.

Un ejemplo de este grado de incerteza se observa en el palacio en el cual se reúnen Juanito y Blanca e inician su historia de amor. El narrador esboza una vaga descripción tanto de sus características como de su localización. Allí llega Juanito siguiendo a Blanca después de descubrirla en la catedral. Como única pista se nos dice que recorre "las revueltas calles de la ciudad" (idem: 41), referencia de carácter genérico, hasta llegar a un antiguo e imponente palacio. Allí se celebrará días más tarde un suntuoso baile ofrecido por "el marqués de B..." (ibidem). De nuevo nos encontramos ante el recurso de la elipsis nominativa tan acorde a la vaguedad discursiva que impregna toda la narración:

Eran las últimas noches del mes de Febrero del año $184 \ldots$ y entre los círculos más animados de la ciudad era público que el marqués de B... daría un suntuoso baile. Todos esperaron esa noche como una noche de felicidad (...). Si atravesáis el portal, si adelantáis más, os hallaréis en un ancho patio, mal empedrado, cubierto de hierbas, y en medio dos acacias sucias, viejas y torcidas, de cuyas ramas penden al sol varios pedazos de lienzo acabados de lavar. (ibidem)

A la hora de discernir si se trata de un espacio asignable o claramente ilocalizable es precisa una labor tanto de exégesis literaria como cartográfica. Pocos son los datos aportados por el texto para proceder al análisis de su incertidumbre, más allá de que consta de numerosos salones y que desde la entrada se vislumbra un patio central con una escalinata al fondo. Por tanto, nuestro punto de partida consta de escasos parámetros: la condición de palacio con un patio interior y soportales enfrente, donde esperaban para entrar en la fiesta "buen golpe de estudiantes, envueltos en sus capas y arrimados a las columnas de los soportales, frente a la casa del marqués de B...." (ibidem). Del mismo modo, 
es necesario considerar si el vínculo del edificio con el "marqués de B..." constituye o no una licencia poética.

Estas escasas referencias exigen un arduo análisis. A partir de los datos espaciales del texto es posible determinar que el palacio debía ser de grandes dimensiones, situarse en el casco histórico de Santiago y en una calle con soportales. El análisis cartográfico resultó clave, puesto que permitió limitar las opciones a tres palacios, partiendo de las variables anteriormente señaladas. Solo dos de ellos, el pazo de Bendaña y el palacio del Marqués de Rivadulla, se ajustan a la descripción geográfica y arquitectónica de la novela, además de coincidir su inicial con la propuesta en la narración: "marqués de B...". El primero, situado en la plaza del Toural, pertenecía a los marqueses de Bendaña, mientras que el segundo, sito en la rúa Nova, era propiedad de la familia Ibáñez de Mondragón, cuyo blasón está formado por dos dragones sujetando la letra "B". Asimismo, ambas construcciones civiles destacaban por sus grandes dimensiones y su escudo de armas en la fachada.

La diferencia clave entre ambos es que únicamente el palacio de la familia de Ibáñez de Mondragón albergaba un patio central. La existencia de un patio central es la única descripción precisa que el narrador apunta del interior del palacio. A este dato revelador se suma la conocida filiación de la familia Ibáñez de Mondragón con la zona del Ulla, escenario clave de la novela Desde el cielo. Allí poseían un célebre pazo, cuyas tierras dieron nombre al marquesado. Por otra parte, está documentado que la familia Ibáñez de Mondragón gozaba de numerosas propiedades, algunas de ellas "desta parte del río de la Ulla hacia la ciudad de Santiago", tal y como se apunta en el tomo III de la Enciclopedia Blasones y linajes de Galicia (1965: 311). En este punto, cabe recordar que Blanca vivía en una quinta a las orillas del Ulla, en el límite con "P...", sugiriendo estos datos espaciales una potencial relación entre la quinta y el palacio.

También resulta reveladora la historia de la estirpe de los Ibáñez Mondragón y su vínculo con la ciudad. La familia Mondragón se asienta en Compostela a finales del siglo XV, procedente del País Vasco, haciéndose rápidamente con un patrimonio artístico y territorial de gran relevancia. Su situación privilegiada en la ciudad se ve reflejada en la construcción de la capilla catedralicia conocida como La Piedad, encargada por el canónigo don Juan de 
Mondragón en 1521 y concluida en apenas cinco años. En línea con un fuerte ascenso social y económico, en 1552 solicitó licencia para reedificar un palacio en la rúa Nova. Décadas más tarde el palacio albergará una notable biblioteca, estudiada por Leopoldo Fernández Gasalla (1995), que muestra la deriva intelectual de la familia.

Por otra parte, este edificio es notablemente más antiguo que el pazo de Bendaña, levantado en el siglo XVIII. Otro elemento a tener en cuenta es que la "B" de Ibáñez no solo aparece en su escudo, sino que se repite en la forja de los balcones que decoran el primer piso de la fachada, a la vista de cualquier viandante. Recordemos que el blasón ornamenta tanto la fachada como el patio interior, donde aparece por duplicado junto al escudo de los Eremuzqueta, orden religiosa con quien estaba emparentada la familia.

En conclusión, esta relación de datos pone de manifiesto la tarea dificultosa, a la par que apasionante, de discernir la incertidumbre de los espacios sin trasunto real, obligando a una exégesis documental, cartográfica y literaria, así como a una arriesgada toma de decisiones.

Otro ejemplo de espacio asignable en segundo grado, donde la cartografía juega un aspecto clave, atañe a la localización de las casas en Padrón de los tres protagonistas. De nuevo, la imprecisión y el protagonismo de la naturaleza dificultan su localización geográfica. La novela empieza con una descripción del Ulla que conduce a la quinta de Blanca. A ella se llega desde "un paseo orillado de acacias" y se sitúa "cerca del lugar en el que el Ulla se ensancha y traspasa los límites de su cauce para entrar con el estrépito de cien armonías en el Océano" (idem: 19). Por su parte, de Marta se dice que "desde su ventana se veía a lo lejos la mar de la ría de Arosa, coqueta y melancólica como una mujer dormida" (idem: 23).

De las tres casas, únicamente resulta ser asignable y, por lo tanto, mapeable, la casa de Marta, pues en el texto se especifica que bajo el arco de su ventana pasa el Ulla y a lo lejos se adivina la ría, datos que favorecen la pesquisa cartográfica. De la residencia de Juanito no se da ninguna referencia, más allá de que está en la propia villa, razón por la cual su vivienda se considera un espacio ilocalizable, pues podría tratarse de cualquier casa de la villa o de ninguna de ellas. Un caso más complejo es la quinta de Blanca, dado que los 
enclaves registrados a través de los Sistemas de Información Geográfica resultan incompatibles con las características espaciales descritas en la novela, pues las quintas estaban emplazadas en zonas altas que orográficamente no son susceptibles de ser inundadas. En consecuencia, no cabe sino resolver que la quinta de Blanca constituye un espacio ilocalizable.

\section{Conclusiones.}

Este trabajo constituye una respuesta en ciernes a una de las lagunas que más debate y expectación en cartografía literaria. En esta novela, la discursivización de la incerteza viene marcada por el aire legendario típico del relato romántico, la fuerza sobrenatural de la naturaleza y la intensificación del espacio. A pesar de las innegables particularidades del texto, nuestro propósito ha sido apostar y arriesgar, por una tipología donde la herramienta cartográfica resulta decisiva a la hora de navegar por la incertidumbre. A través del estudio de caso que favorece la novela Desde el cielo de Manuel Murguía, hemos podido elaborar una caracterización de incertidumbre espacial que se adapta a la especificidad del discurso literario propio de esta obra. A partir de dicho paradigma, nos ha sido posible desarrollar una tipología de espacios apta para pautar los diferentes tipos de incertidumbre espacial discursiva en función de los métodos de análisis de la cartografía literaria y de los Sistemas de Información Geográfica empleados en cada caso. Esta clasificación ha permitido hallar el referente real de los principales espacios que soportan la trama de la obra, neutralizando la imprecisión y vaguedad características de la narración romántica, tradición en la que Desde el cielo se inscribe. 


\section{NOTAS}

${ }^{1}$ Este trabajo se enmarca en el proyecto "La proyección del lugar: Compostela en su imaginario geoliterario (18441926). Sistemas de Información Geográfica y humanidades espaciales", financiado por el Ministerio español de economía y competitividad (FFI2013-41361-P). En el marco de este proyecto y al amparo económico de la misma institución, Alba Rozas Arceo es beneficiaria del contrato predoctoral BES-2014-068528 para llevar a cabo la tesis "Fragmentos de Apocalipsis. La proyección cartográfica de Santiago de Compostela a partir del imaginario geoliterario" cuyo marco teórico entronca con el de la presente investigación.

${ }^{2}$ Manuel Murguía (1833-1923) fue una de las figuras claves del Rexurdimento. Historiador, escritor y creador de la Real Academia Galega, destacó a lo largo de su vida por su compromiso hacia la cultura y la historia gallega, incidiendo en la importancia de su divulgación.

${ }^{3}$ El atlantismo defiende el vínculo de la cultura gallega con los pueblos irlandeses, normandos y bretones. Aunque ya encontramos voces como la de Murguía que postulan esta teoría, habrá que esperar a la llegada de Vicente Risco y la Xeración Nós para que goce de mayor difusión. 


\section{Bibliografía}

Auger, A./ J. Roy (2008), "Expression of Uncertainty in Linguistic Data", 11 ${ }^{\text {th }}$ International Conference on Information Fusion, Colonia, Alemania, pp. 1860-1867. Recuperado de: http://fusion.isif.org/proceedings/fusion08CD/papers/1569107673.pdf?.

Blasones y linajes de Galicia (1965), vol III, Santiago de Compostela, Editorial de los Bibliófilos Gallegos.

Buttenfield, B. (1993), “Representing data quality”, Cartographica, vol. 30, no 2\&3, pp. 1-7.

Cabo Aseguinolaza, F. (2016), “Topografía y alegoresis en El primer loco, de Rosalía de Castro", Revista de Estudios Hispánicos, 50, pp. 220-241.

Castro, R. de (1993a), Obras completas de Rosalía de Castro, 2 vols., Madrid, Turner.

-- (2004b), Follas novas, Vigo, Galaxia [1880].

Drecki, I., (2007), “Geographical information uncertainty: The concept and representational challenges", Proceedings of the 23 $3^{\text {rd }}$ International Cartographic Conference, Moscú, Rusia [sin paginar]. Recuperado de: http://icaci.org/files/documents/ICC_proceedings/ ICC2007/abstracts/html/1_Oral3-1_3_GEOGRAPHICAL\%20INFORMATION\% 20UNCERTAINTY.htm

Egenhofer, M. J./ D. M. Mark (1995), "Naive geography”, Lecture Notes in Computer Science, in A. Frank and W. Kuhn (eds.) (1995), Lecture Notes in Computer Science, vol. 988, Springer-Verlag, pp. 1-15.

Fernández Gasalla, L. (1995), “La Biblioteca de D. Andrés de Mondragón, I Marqués de Santa Cruz de Rivadulla, mecenas y político gallego del siglo XVII (1645-1709)", Cuadernos de Estudios Gallegos, tomo XLII, fascículo 107, pp. 499-564.

Griethe, H./ H. Schuman (2006), "The visualization of uncertain data: methods and problems", Proceedings of simulation and visualization 2006, Magdeburg, Alemania [sin paginar]. Recuperado de: https://www.researchgate.net/publication/216756553

N.. 38 - 6/ 2018 | 23-44 - ISSN 2183-2242 | http:/dx.doi.org/10.21747/21832242/litcomp38a2 


\section{The Visualization of Uncertain Data Methods and Problems}

MacEachren, A. M. et al. (2005), "Visualizing geospatial information uncertainty: what we know and what we need to know", Cartography and Geographic Information Science, no 32, pp. 139-160.

Murguía, M. (1888a), Galicia, Barcelona, Establecimiento tipográfico-editorial de Daniel Cortezo y Cia.

-- (1910b), Desde el cielo, Madrid, Imprenta artística española. Recuperado de: http://biblioteca.galiciana.gal/pt/consulta/registro.cmd?id=8360

Reuschel, A. K./ L. Hurni (2011), “Mapping Literature: Visualisation of Spatial Uncertainty in Fiction", The Cartographic Journal, vol. 48, no 4, pp. 293-308.

Shu, H. et al. (2003), Uncertainty of Geographic Information and Its Support in MADS, $2^{\text {nd }}$ International Symposium on Spatial Data Quality, Hong Kong, China, pp. 1-13. Recuperado de:https://www.researchgate.net/publication/37442688_Uncertainty_of_Geographic_Info rmation_and_its_Support_in_MADS

Thomson, J. et al. (2005), "A Typology for Visualizing Uncertainty", Proceedings of the IS\&T/SPIE Symposium on Electronic Imaging, San Jose, CA USA [sin paginar]. Recuperado de:https://www.researchgate.net/publication/216756513_A_Typology_for_Visualizing_U ncertainty

Waters, T./ A. J. Evans (2003), “Tools for web-based GIS mapping of a 'fuzzy' vernacular geography", Proceedings of the $7^{\text {th }}$ International Conference on Geocomputation, Leeds, Inglaterra [sin paginar]. Recuperado de:http://www.geocomputation.org/2003/Papers/ Waters_Paper.pdf

Zhang, J./ M. F. Goodchild (2002), Uncertainty in geographical information, New York, Taylor\&Francis. 
Arantxa Fuentes Ríos es Doctora europea en Literatura comparada y Teoría de la literatura y actualmente profesora en la Universidad de Santiago de Compostela. La mayor parte de su carrera docente ha sido desarrollada en el extranjero, en la University of Richmond (VA, EEUU) y la Université de la Bourgogne (Dijon, Francia). Antigua becaria de la Casa de Velázquez, ha publicado diversos artículos en revistas nacionales e internacionales sobre teoría de la historia literaria. Su línea principal de investigación está ligada a la figura del poeta-crítico, en concreto Antonio Machado, José Ángel Valente u Octavio Paz. Pertenece al equipo del proyecto subvencionado por el Ministerio de Educación y Ciencia titulado La proyección del espacio: Compostela en su imaginario geoliterario. Sistemas de Información Geográfica y Humanidades espaciales.

\begin{abstract}
Alba Rozas Arceo es licenciada en Filología Gallega y Filología Hispánica por la Universidade de Santiago de Compostela y becaria predoctoral del proyecto "La proyección del lugar: Compostela en su imaginario geoliterario. Sistemas de Información Geográfica y Humanidades Espaciales". En el marco de este proyecto lleva a cabo la tesis "Fragmentos de Apocalipsis. La proyección cartográfica de Santiago de Compostela a partir del imaginario geoliterario". Cuenta con artículos especializados en el ámbito de la geografía literaria y los imaginarios espaciales como «El Hobbit: una aventura cartográfica» publicado en el nำ1 de Elos. Revista de Literatura Infantil e Xuvenil y «The Hobbit desde a xeografía literaria crítica. Perspectivas de análise» en el n047 del Boletín Galego de Literatura.
\end{abstract}

